\title{
Práticas e saberes sobre doenças infecciosas e parasitárias transmitidas por mosquitos vetores entre escolares de Maceió, Alagoas
}

Practices and knowledges about infectious and parasitic diseases transmitted by mosquitoes among schoolchildren in Maceió city, Alagoas state

Prácticas y conocimiento sobre enfermedades infecciosas y parasitarias transmitidas por mosquitos vectores entre escolares de Maceió, Alagoas

Recebido: 10/09/2021 | Revisado: 16/09/2021 | Aceito: 21/09/2021 | Publicado: 21/09/2021

Karwhory Wallas Lins da Silva ORCID: https://orcid.org/0000-0001-7015-2489

Centro Universitário Cesmac, Brasil

E-mail: yrohwrak@outlook.com

Daniela Calumby de Souza Gomes ORCID: https://orcid.org/0000-0003-0079-793X

Centro Universitário Cesmac, Brasil

E-mail: calumby_biomed@ @otmail.com

Janaila Cristina Braz da Silva

ORCID: https://orcid.org/0000-0001-9888-8068

Centro Universitário Cesmac, Brasil

E-mail: janailacb@outlook.com

Taynarianne Ferreira Alves

ORCID: https://orcid.org/0000-0003-0685-734X

Centro Universitário Cesmac, Brasil

E-mail: taynarianne@hotmail.com

Kézia Kewyne Lins da Silva

ORCID: https://orcid.org/0000-0002-5645-1586

Centro Universitário Cesmac, Brasil

E-mail: keziakw8@gmail.com

Jéssica Alves Silva Oliveira

ORCID: https://orcid.org/0000-0002-7116-6476 Centro Universitário Cesmac, Brasil E-mail: jessicaaso@hotmail.com

Sâmea Keise de Oliveira Silva

ORCID: https://orcid.org/0000-0002-8447-6326 Centro Universitário Cesmac, Brasil E-mail: samea-keyse@ hotmail.com

Susane Maria de Santana Barros ORCID: https://orcid.org/0000-0002-9333-256X Centro Universitário Cesmac, Brasil E-mail: susanebarros@hotmail.com

Ana Katariny de Barros Silva ORCID: https://orcid.org/0000-0002-1659-3635 Centro Universitário Cesmac, Brasil E-mail: katarinybarros@hotmail.com

Lindon Johoson Diniz Silveira ORCID: https://orcid.org/0000-0002-6584-4568 Centro Universitário Cesmac, Brasil E-mail: johoson@uol.com

Saskya Araújo Fonseca ORCID: https://orcid.org/0000-0003-2091-7951 Centro Universitário Cesmac, Brasil E-mail: saskya_mcz@hotmail.com

Thiago José Matos-Rocha

ORCID: https://orcid.org/0000-0001-5153-6583 Universidade Estadual de Ciências da Saúde de Alagoas, Brasil E-mail: tmatosrocha@cesmac.edu.br

\section{Resumo}

As Doenças Infecciosas e Parasitárias (DIPs) são um problema de saúde pública mundial. No estado de Alagoas, a presença de muitos rios, riachos, lagos e lagoas favorece a proliferação dos mosquitos. Neste trabalho, buscou-se verificar as práticas e saberes sobre DIPs transmitidas por mosquitos vetores (Aedes sp., Lutzomya sp., Anopheles sp. 
e C. quinquefasciatus) entre estudantes de uma comunidade escolar de Maceió, Alagoas. Foi aplicado um questionário sobre as DIPs e seus mosquitos vetores para os alunos do ensino médio de uma escola estadual. Posteriormente, foram realizadas palestras sobre essas doenças e também uma oficina de construção da mosquitérica ${ }^{\circledR}$, além da exibição de um documentário da Fiocruz. Entre os alunos, 57,34\% eram do sexo feminino e 42,66\% do masculino; a faixa etária variou de 14 a 22 anos. Verificou-se que 97,90\% do lixo residencial era levado ao aterro sanitário; 98,60\% dos estudantes sabiam que eliminar água parada é importante para combater os mosquitos e 39,86\% acreditavam que a limpeza evita focos dos mosquitos. Durante as ações, os estudantes se demonstraram interessados em aprender sobre as consequências das doenças e como evitar a proliferação dos mosquitos. Os resultados expõem que os alunos sabem da importância de evitar os mosquitos e também o que devem fazer para evitar a proliferação dos mosquitos vetores. Eles podem, portanto, ser multiplicadores de boas práticas de saúde e ambientais.

Palavras-chave: Infecções por arbovirus; Doenças parasitárias; Dípteros; Educação em saúde; Educação em saúde ambiental.

\begin{abstract}
Infectious and Parasitic Diseases (IPDs) are a major global public health problem. The presence of many rivers, streams, lakes and lagoons is conducive to the proliferation of mosquitoes in Alagoas State. This study aims to analyse practices and knowledges about IPDs transmitted by mosquitoes (Aedes sp., Lutzomya sp., Anopheles sp. and C. quinquefasciatus) among students from a school-community in Maceió, Alagoas. A survey instrument of IPDs and their vector mosquitoes was applied for high school students from a state school. Later, lectures on the diseases were given, a workshop for fabrication of mosquito nets was offered and a documentary by Fiocruz was shown. Regarding to the students, $57.34 \%$ were females and $42.66 \%$ were males, age range 14 to 22 years-old. The results indicate that $97.90 \%$ of the waste generated in their homes is transported to the landfill; $98.60 \%$ of the students knew the importance of eliminating standing water to fight mosquitoes; and $39.86 \%$ of the participants believed that a cleaned house avoids places where the mosquito lays its eggs. During this process, students were interested in learning about the consequences of diseases and ways of preventing mosquito proliferation. The data reveals that students know the importance of avoiding mosquitoes and also know what to do to prevent the proliferation of vector mosquitoes. Therefore, they can be multipliers in order to increase good health and environmental practices.
\end{abstract}

Keywords: Arbovirus infections; Parasitc diseases; Diptereas; Health education; Environmental health education.

\title{
Resumen
}

Las Enfermedades Infecciosas y Parasitarias (EIPs) son un problema de salud pública mundial. En el Estado de Alagoas, la presencia de numerosos ríos, arroyos, lagos y lagunas favorece la proliferación de mosquitos. En este trabajo se buscó verificar las prácticas y conocimientos sobre las EIPs transmitidas por mosquitos vectores (Aedes sp., Lutzomya sp., Anopheles sp. y C. quinquefasciatus) entre estudiantes de una comunidad escolar. Se aplicó un cuestionario sobre EIPs y sus mosquitos vectores a estudiantes de seundaria en unal escuela estatal. Se llevaron a cabo conferencias posteriores sobre estas enfermedades y también un taller sobre la construcción de la mosquitera, además de la exhibición de un documental de la Fiocruz. Entre los estudiantes, el 57,34\% eran mujeres y el 42,66\% hombres; el rango de edad osciló entre 14 y 22 años. Se encontró que el $97.90 \%$ de los residuos residenciales fueron llevados al relleno sanitario; $98,60 \%$ de los estudants sabían que eliminar el agua estancada es importante para combatir los mosquitos y el 39,86\% de los participantes creía que la limpieza previene los brotes de mosquitos. Durante las acciones, los estudiantes mostraron interés en conocer las consecuencias de las enfermedades y cómo prevenir la proliferación de mosquitos. Los resultados muestran que los estudiantes conocen la importancia de evitar los mosquitos y también lo que deben hacer para prevenir la propagación de los mosquitos vectores. Por tanto, pueden ser multiplicadores de buenas prácticas sanitarias y medioambientales.

Palabras clave: Infecciones por arbovirus; Efermedades parasitarias; Dipteranos; Educación para la salud; Educación para la salud ambiental.

\section{Introdução}

Doenças Infecciosas e Parasitárias (DIPs) fazem parte de um grande grupo de doenças causadas por vírus, bactérias e fungos ou protozoários e helmintos; ainda são responsáveis por problemas de saúde pública em todo o mundo, provocando alto índice de morbi-mortalidade, principalmente em países subdesenvolvidos (Ministério da Saúde, 2010).

Atualmente, as doenças transmitidas por vetores biológicos vêm ganhando proporções difíceis de estimar, representando um desafio que exige novas estratégias de prevenção, combate e controle que eliminem ou minimizem essa prevalência e/ou incidência, já que muitos métodos existentes não têm alcançado êxito (Oliveira, Fernandes, \& Moura, 2012).

As principais Doenças Tropicais Negligenciadas (DTNs), no Brasil, transmitidas por mosquitos vetores da ordem Diptera são: dengue, zika, chikungunya, febre amarela, leishmaniose, malária e filariose linfática. Não se tem, por enquanto, 
grandes investimentos para erradicação e controle dessas doenças (Valverde, 2016).

Os mosquitos do gênero Aedes (família Culicidae) são facilmente encontrados e amplamente conhecidos no Brasil por transmitirem o vírus da dengue (Dengue virus (DENV)). Nos últimos anos, surgiram os casos de zika e chikungunya, cujos arbovírus Zika virus (ZIKV) e Chikungunya virus (CHIKV), respectivamente, passaram a ser transmitidos também pelo Aedes. Os sinais e sintomas dessas três doenças são semelhantes entre si: febre, cefaleia, artralgia e prurido, que duram de 2 a 10 dias (Souza Neto, 2016).

Outra doença já conhecida por ser transmitida pela picada do Aedes aegypti é a febre amarela, encontrada no Norte e Nordeste do Brasil, causada por vírus da família Flaviviridae, do gênero Flavivírus, apresentando formas clínicas que variam de assintomáticas até graves, com síndromes ictero-hemorrágicas e hepato-renal (Mascheretti et al., 2013).

A leishmaniose foi a primeira zoonose de grande impacto, transmitida por mosquito, a ser investigada. Essa prioridade se deu devido à dificuldade de tratamento e evolução rápida para a forma mais grave da doença. Os mosquitos do gênero Lutzomyia pertencem à família Psychodidae, e transmitem os parasitos do gênero Leishmania, que provocam manifestações cutâneas e viscerais, em muitas pessoas de diferentes regiões do Brasil e do mundo (Queiroz, Varjão, Moraes, \& Salcedo, 2012).

A Leishmaniose Tegumentar Americana (LTA) é caracterizada por lesões cutâneas únicas, múltiplas e indolores nos seres humanos e possuem implicações epidemiológicas importantes. Em outros tempos, considerada uma endemia rural, expandiu-se para regiões urbanas do Brasil com registro de 28.68 casos autoctones. A Leishmaniose Visceral Americana (LVA) apresenta-se como a forma mais grave da doença, sendo considerada emergente em várias partes do mundo, incluindo 12 países da América Latina, devido ao aumento gradativo dos problemas socioambientais ao longo dos anos (Cordeiro, Silva, \& Oliveira, 2015).

Para além do gênero Aedes e Lutzomyia, existem também outros dípteros, como os do gênero Anopheles e o Culex quinquefasciatus, que são vetores da malária e da filariose linfática, respectivamente. A malária é uma doença febril causada, no Brasil, pelos protozoários Plasmodium falciparum, P. vivax e $P$. ovale, para a qual não há, ainda, vacinas, devendo, portanto, ser adotadas medidas que evitem a picada do mosquito (Braga \& Fontes, 2010).

A filariose linfática é causada pelo helminto Wuchereria bancrofti, que atinge áreas urbanizadas e provoca diversas deformidades e incapacidades nos indivíduos por ela acometidos; sua incidência está relacionada a fatores socioambientais, como falta de saneamento básico e de coleta de lixo (Braga et al., 2001). Embora já tenha sido eliminada em quase todos os estados brasileiros, ainda é uma doença endêmica em Pernambuco, necessitando de ações de prevenção, tratamento precoce e controle (Aguiar-Santos et al., 2013).

É importante lembrar que os mosquitos se proliferam utilizando água parada, que se acumula em recipientes como garrafas e embalagens descartáveis, latas, pneus, entre outros artefatos, usados em atividades do dia-a-dia. Além da população descartar o lixo de forma inadequada, outros fatores favorecem a presença dessas epidemias no Brasil, como: o clima tropical, as florestas tropicais e o rápido crescimento demográfico associado à intensa e desordenada urbanização (Confalonieri, 2003).

Em Maceió, o crescimento populacional desordenado como, por exemplo, a ocupação das encostas nas margens do Riacho Reginaldo, tem causado impactos ambientais importantes: habitações impróprias, acúmulo de lixo e inundações que podem favorecer a proliferação dos mosquitos e afetar a saúde da população (Pedrosa, 2008). Por essa razão, é preciso que as ações de controle exijam não só a participação ativa de diferentes setores da administração pública mas também a participação efetiva de cada morador na eliminação de criadouros já existentes ou de possíveis locais para reprodução dos mosquitos.

Diante de tais fatos, este trabalho teve como finalidade verificar as práticas e saberes sobre DIPs transmitidas por mosquitos vetores (Aedes sp., Lutzomya sp., Anopheles sp. e C. quinquefasciatus) entre estudantes de uma comunidade escolar de Maceió, Alagoas, viabilizando o desenvolvimento de novas estratégias no combate a estes vetores. 


\section{Metodologia}

\subsection{Aspectos éticos}

Este trabalho foi submetido ao Comitê de Ética e Pesquisa (CEP) do Centro Universitário Cesmac, sob o Certificado de Apresentação e Apreciação Ética (CAAE) de número: 67287317.8.0000.0039. E aprovado sob o número: 2.080.261.

\subsection{Tipo e local de estudo}

Foi realizado um estudo descritivo, transversal, de caráter quali-quantitativo. O estudo foi realizado em uma escola estadual, localizada na cidade de Maceió, Alagoas. Sendo desenvolvido com todas as turmas do ensino médio em duas etapas: coleta e análise das informações; e benefícios para os voluntários.

\subsection{Coleta e análise das informações}

Para a execução do projeto, inicialmente, foram apresentados à direção da escola estadual, a importância e o objetivo da pesquisa, a fim de requerer a permissão para convidar os alunos das turmas acima citadas a participarem do desenvolvimento desta pesquisa.

Os critérios de inclusão foram: serem estudantes regularmente matriculados no ensino médio, que aceitassem participar da pesquisa, o que seria comprovado pela assinatura dos termos do CEP. Os critérios de exclusão foram aqueles que não estiveram presentes no dia ou que não assinaram o Termo de Consentimento Livre e Esclarecido (TCLE) ou Termo de Assentimento Livre e Esclarecido (TALE). A abordagem aos alunos para aplicação dos questionários foi realizada diretamente na escola onde estudavam, no período de julho a outubro de 2017.

A utilização do questionário como técnica de investigação é proposta por Gil (2008), onde este apresenta algumas vantagens deste recurso, como por exemplo: possibilidade de atingir grande número de pessoas; garantia do anonimato das respostas; permite que as pessoas respondam quando julgarem mais oportuno; e não expõe os pesquisados à intervenção do entrevistador.

O TCLE foi entregue para os alunos a partir de 18 anos de idade e o TALE para os que não atingiram a maior idade, bem como, o TCLE para o seu responsável. Mediante a assinatura destes documentos, após serem recrutados através da palestra de sensibilização, os alunos foram convidados a preencher o instrumento de coleta de dados - questionário -, sem interferência de outrem, baseado no estudo de Cordeiro, Silva e Oliveira (2015), em sala reservada cedida pela diretoria da escola. As perguntas foram formuladas de forma objetiva, com adequação da linguagem para facilitar a compreensão do que se perguntava, incluindo-se, inicialmente, questões referentes aos dados socioeconômicos (sexo, idade, renda e moradia).

A segunda parte do questionário contemplou aspectos relacionados aos fatores envolvidos na transmissão das DIPs: métodos de prevenção das DIPs, existência de fossa sanitária no domicílio; criação de animais e presença de mosquitos na residência, horário com maior concentração de mosquitos, conhecimento sobre arboviroses e parasitoses, visitação de agentes de endemias, se já foram acometidos por alguma DTN, entre outros.

A análise dos dados foi realizada de maneira descritiva com embasamento teórico-científico, sendo realizada a seleção, focalização, abstração e a transformação destes por meio de estatística simples considerando a frequência absoluta (n) e relativa (\%). Utilizou-se o software Microsoft Office Excel $^{\circledR}$ (Gil, 2008; Ludke \& Andre, 2013; Pereira, Shitsuka, Parreira, \& Shitsuka, 2018).

\subsection{Benefícios para os voluntários}

$\mathrm{Na}$ segunda etapa deste trabalho, foram produzidos materiais didáticos para realização das palestras in loco sobre dengue, zika, chikungunya, febre amarela, leishmaniose, malária e filariose linfática, apresentando os agentes causadores, formas de transmissão, sintomatologia, formas de prevenção e controle (Cordeiro, Silva, \& Oliveira, 2015). Posteriormente, foi 
elaborado um fluxograma para auxiliar durante a execução da oficina de construção da mosquitérica ${ }^{\circledR}$, um dispositivo ecológico e econômico, produzido com garrafas de Politereftalato de Etileno (PET) (Faperj, 2017). E por fim, o "cinepipoca" idealizado para facilitar o entendimento sobre a dispersão das espécies dos mosquitos, através da exibição de um documentário (Cordeiro, Silva, \& Oliveira, 2015).

\section{Resultados e Discussão}

\subsection{Informações coletadas}

Preencheram os questionários 143 alunos das $1^{\mathrm{a}}, 2^{\mathrm{a}}$ e $3^{\mathrm{a}}$ séries do ensino médio, 57,34\% eram do sexo feminino e $42,66 \%$ do sexo masculino, com idade variando entre 14 a 22 anos e renda familiar predominantemente entre 1 e 2 salários mínimos. As famílias com renda per capita inferior a um salário mínimo perfizeram apenas o mínimo dos registros. Com relação à moradia, 74,82\% dos alunos residiam em casa própria e, em sua maioria, com mais três pessoas.

Em Alagoas, uma pesquisa realizada em 274.059 domicílios constatou que, deste total, 186.647 são próprios, 72.707 alugados, 13.572 cedidos e 1.133 correspondem a outras formas de ocupação. O que demonstrou que a proporção de domicílios próprios é grande (Anuário Estatístico de Alagoas, 2017).

Doenças transmitidas por mosquitos afetam desproporcionalmente a maioria menos privilegiada da população brasileira, de várias maneiras. A crise atual é indicativa da disparidade existente não só em termos de classes sociais mas também de uma variedade de outras questões ligadas à estrutura dessas classes (Lesser \& Kitron, 2016).

Em pesquisa realizada em município do Mato Grosso no ano de 2010, foram registrados 391 casos de dengue, com predomínio de vítimas do sexo masculino $(51,4 \%)$ - a dengue clássica foi o tipo mais prevalente. A faixa etária mais acometida foi de 20 a 34 anos (Assunção \& Aguiar, 2014).

No Nordeste, o vírus da zika teve impacto maior na Bahia, Pernambuco e Rio Grande do Norte, estados em que a percentagem maior da população é pobre e as condições climáticas são mais favoráveis à propagação dos vírus transmitidos por mosquitos, diferentemente de alguns estados da região Sul, com índice de pobreza menor e clima menos tropical (Lesser \& Kitron, 2016).

Alguns fatores repercutem diretamente na incidência dos casos de infecções por parasitos e até mesmo na proliferação de mosquitos vetores, entre os quais é possível citar as condições precárias de saneamento básico, ausência de higiene pessoal e até doméstica, presentes, com maior frequência - ou intensidade - em domicílios com renda per capita de um salário mínimo ou menos que isso. Não se pode desconsiderar também a falta de uma política de educação em saúde comprometida com a diminuição dos casos de doenças transmitidas por mosquitos; tanto das parasitoses, quanto dos mosquitos vetores.

Com relação à presença de fossa sanitária em suas residências, 62,93\% dos participantes responderam que sim, enquanto que 34,97\% responderam que não; $97,90 \%$ do total de alunos que responderam ao questionário disseram que têm acesso aos serviços de aterro sanitário, o que indica um ponto positivo de bastante relevância, já que o lixo acumulado é responsável pela transmissão de doenças, por abrigar vetores que ali encontram alimentos e condições adequadas para proliferação (Ribeiro \& Rooke, 2010). Comparando-se estes índices com o estudo de Cordeiro, Silva e Oliveira (2015), realizado no município de Viçosa, Alagoas, nota-se um resultado inferior (90,50\%) e superior (91,50\%), respectivamente.

De acordo com a Agência Brasil, surtos de dengue no verão estão associados às deficiências de saneamento básico. Esta é uma informação importante, já que existe ainda um percentual importante de pessoas que não dispõe do serviço de fossa sanitária, sendo esta de grande relevância à saúde, uma vez que, não havendo tal estrutura, o ambiente doméstico passa a ser propício à proliferação de doenças, ou seja, um perigo para o bem-estar da população (Laboissière, 2016).

Um dos grandes problemas ambientais na atualidade está voltado para o consumo desenfreado da população e a consequente geração de resíduos. Estima-se em cerca de trinta bilhões de toneladas o total de resíduos sólidos anuais gerados 
pelos seres humanos (Borges \& Oliveira, 2011).

Uma minoria da população ainda mantém o precário hábito de queimar ou enterrar o lixo. Os dados do Anuário Estatístico de Alagoas (2017) informam que, de 1.051 domicílios, 754 recebem a coleta diretamente, enquanto 297 fazem uso de coleta indireta, entre outros artifícios de descarte do lixo. Esses dados já representam mais um ponto positivo para a prevenção de doenças.

O saneamento básico no Brasil é caracterizado por grande desigualdade ao acesso, em relação à coleta e tratamento do esgoto. A disponibilidade do saneamento básico interfere na qualidade de vida da população, afetando principalmente a saúde (Silva, Gama, Callado, \& Souza, 2017).

Segundo a Organização Mundial de Saúde (OMS), saneamento é o controle de todos os fatores do meio físico do homem, que exercem ou podem exercer efeitos nocivos sobre o bem estar físico, mental e social. O sistema de esgoto existe para afastar a possibilidade de contato de dejetos humanos com a população, com as águas de abastecimento, com os vetores de doenças e com os alimentos (Ribeiro \& Rooke, 2010).

Na Tabela 1 vê-se os métodos preventivos, conhecimento e hábitos sobre os mosquitos. Os métodos preventivos mais adotados pelos estudantes foram, em sua maioria, a eliminação de água parada e a não acumulação de lixo, com um percentual de $81,81 \%$ e $61,53 \%$, respectivamente, o que demonstra um ponto derterminante no que se refere ao conhecimento dos estudantes. Este dado é refletido positivamente na questão posterior que pergunta sobre a presença do mosquito nas residências. Sobre isso, 56,64\% relataram a presença de poucos mosquitos, sendo a sua prevalência maior $(69,93 \%)$ no turno da noite.

Os dados da Secretaria de Estado da Saúde de Alagoas mostram que se comparando os anos de 2016 e 2017, a transmissão de arboviroses pelo mosquito sofreu uma redução: dengue (94,29\%), zika (98,76\%) e chikungunya (89,50\%). Isso demonstra a efetividade e positividade das campanhas de prevenção dos mosquitos vetores (Sesau, 2017).

Através da Tabela 1 também é possível verificar que a maior taxa de conhecimento sobre as doenças transmitidas por mosquitos foi relacionada a chikungunya $(97,20 \%)$, seguida da dengue $(96,50 \%)$ e zika $(95,50 \%)$. Estas doenças tornaram-se populares devido aos surtos e às estratégias de prevenção noticiados na mídia local. Apenas uma minoria citou a leishmaniose (8,39\%), percebendo-se que os alunos não têm conhecimento sobre tal patologia, o que demanda preocupação, pois a LVA é influenciada por fatores relacionados às condições de criação de animais nos domicílios, em especial o cão. É necessário que se esclareça para a população o risco do convívio tão próximo e cada vez mais estreitado entre o homem e seus animais domésticos (Borges et al., 2009).

Nesta pesquisa pesquisa foi constatado que 53,85\% dos participantes criavam algum tipo de animal doméstico - em sua maioria, cães (39,86\%). Este resultado se assemelha ao do estudo de Cordeiro, Silva e Oliveira (2015) realizado no interior do estado de Alagoas. Os cães são potenciais geradores da propagação da LVA, seja como atrativa fonte alimentar para o vetor ou pelo potencial de servir como reservatório do parasito (Borges et al., 2009).

A LVA é uma enfermidade infecciosa generalizada, de caráter zoonótico, que provoca, nos indivíduos por ela afetados, febre irregular, hepatoesplenomegalia, emagrecimento, edema e anemia. Sua taxa de letalidade chega a $10 \%$, em casos de tratamento inadequado (Alvarenga, Escalda, Costa, \& Monreal, 2010).

Foi observado um percentual de conhecimento elevado entre os alunos quanto ao questionamento sobre a importância de eliminar água parada (Tabela 1). A maioria respondeu de forma positiva, 98,60\%, indicando sua sensibilização quanto à importância de se ter os cuidados necessários com relação aos mosquitos causadores das DIPs. Este resultado é superior aos detectados por Roriz, Peres e Ramos (2016), que concluíram essa mesma percepção em 86\% dos estudantes no $9^{a}$ ano do ensino fundamental em uma escola no município de Humaitá, Amazonas.

No Brasil, o controle dessas doenças é essencial para a saúde pública, já que ocorrem surtos com certa frequência, 
necessitando-se que seja criada uma cultura de eliminação da água parada para o controle do vetor.

A importância da eliminação de água parada se faz necessária, visto o Aedes aegypti como principal vetor responsável pela transmissão da dengue, zika e chikungunya. Um mosquito de hábitos doméstico e diurno, utilizando-se preferencialmente de depósitos de água limpa para deposição dos ovos (Claro, Tomassini, \& Rosa, 2004).

Tabela 1. Prevenção, conhecimento e hábitos dos mosquitos vetores relatados por estudantes de uma escola estadual de Maceió, Alagoas.

\begin{tabular}{|c|c|c|}
\hline Quais os métodos preventivos você prefere adotar contra os mosquitos? & $\%$ & $\mathbf{n}$ \\
\hline Roupas, meias e luvas & 20,27 & 29 \\
\hline Inseticida & 42,65 & 61 \\
\hline Raquete elétrica & 27,27 & 39 \\
\hline Eliminar a água parada & 81,81 & 117 \\
\hline Mosqueteiro & 37,76 & 54 \\
\hline Não acumular lixo & 61,53 & 88 \\
\hline Não uso método preventivo & 48,95 & 70 \\
\hline Repelente & 4,89 & 7 \\
\hline Não desejo responder & 0,69 & 1 \\
\hline Foi observada a presença de mosquitos em sua residência? & $\%$ & $\mathbf{n}$ \\
\hline Não & 13,28 & 19 \\
\hline Poucos & 56,64 & 81 \\
\hline Intermediário & 12,58 & 18 \\
\hline Muitos & 14,00 & 20 \\
\hline Não desejo responder & 3,50 & 5 \\
\hline Se sim, quais os maiores horários de concentração dos mosquitos na residência? & $\%$ & n \\
\hline Manhã & 2,10 & 3 \\
\hline Tarde & 9,09 & 13 \\
\hline Noite & 69,93 & 100 \\
\hline Três turnos & 4,20 & 6 \\
\hline Não desejo responder & 14,68 & 21 \\
\hline Quais são as doenças transmitidas por mosquitos? & $\%$ & $\mathbf{n}$ \\
\hline Leishmaniose & 8,39 & 12 \\
\hline Filariose linfática & 7,69 & 11 \\
\hline Tricomoníase & 1,39 & 2 \\
\hline Doenças de Chagas & 8,39 & 12 \\
\hline Malária & 27,27 & 29 \\
\hline Câncer & 0,69 & 1 \\
\hline AIDS & 2,09 & 3 \\
\hline Zika & 95,50 & 138 \\
\hline Chikungunya & 97,20 & 139 \\
\hline Dengue & 96,50 & 138 \\
\hline Pneumonia & 1,39 & 2 \\
\hline Não desejo responder & 0,00 & 0 \\
\hline Eliminar água parada é importante no combate os mosquitos? & $\%$ & $\mathbf{n}$ \\
\hline Sim & 98,60 & 141 \\
\hline Não & 0,00 & 0 \\
\hline Não desejo responder & 1,40 & 2 \\
\hline
\end{tabular}

Fonte: Elaboração própria (2017). 
Na Tabela 2, o questionamento foi feito sobre o conceito de arboviroses e parasitoses. Do total, 57,34\% e 77,62\%, responderam que são doenças transmitidas por vírus e parasitos, respectivamente, ratificando mais uma vez um grau considerado de conhecimento em relação às doenças. Em relação ao grau de participação da administração pública na contenção da disseminação dos mosquitos, 50,35\% responderam que sim, confirmando que essa luta precisa abranger todas as esferas da sociedade, incluindo o poder público. Quando questionados sobre se poderiam ajudar o país a combater a proliferação dos mosquitos, felizmente $88,81 \%$ afirmaram que sim, provando que os mesmos pretendiam contrubuir com o combate e controle das doenças transmitidas por mosquitos vetores.

Na Tabela 2 também é possível verificar que infelizmente 39,86\%, acreditavam que a casa limpa evita focos dos mosquitos. É importante enfatizar que para evitar a proliferação dos mosquitos, é fundamental a mobilização comunitária para a adoção de práticas de redução dos criadouros dos mosquitos vetores (Castro \& Queiroz, 2013). As abordagens baseadas na participação comunitária e educação em saúde têm sido cada vez mais valorizadas, ao lado das ações ambientais e da vigilância epidemiológica, entomológica e viral (Claro, Tomassini, \& Rosa, 2004).

Deve-se destacar, no entanto, que não somente à comunidade deve ser atribuída total responsabilidade para o combate ao vetor. Os setores públicos devem estar envolvidos na ação, possibilitando o melhoramento nas condições de urbanização e habitação, a partir de coleta regular de lixo, abastecimento permanente de água encanada e educação escolar direcionada também para esse fim. Com essa atuação conjunta as medidas de prevenção poderiam ter um efeito satisfatório (Lobo, Bezerra, Brito, Silva, \& Pinheiro, 2013).

Sem dúvida, para a diminuição dos casos de doenças transmitidas por mosquitos, é necessário promover melhorias nas condições socioeconômicas, no saneamento básico e na educação sanitária, além de mudanças de certos hábitos culturais da população (Beinner, Norton, \& Lamounier, 2006). 
Tabela 2. Conhecimento sobre DIPs dos alunos de uma escola estadual de Maceió, Alagoas.

\begin{tabular}{l|c|c}
\hline O que são arboviroses? & $\mathbf{\%}$ & $\mathbf{n}$ \\
\hline Doenças transmitidas por vírus & 57,34 & 82 \\
\hline Doenças transmitidas por bactérias & 2,09 & 3 \\
\hline Doenças transmitidas por parasitos & 13,28 & 19 \\
\hline Doenças transmitidas por fungos & 0,69 & 1 \\
\hline Não desejo responder & 26,60 & 38 \\
\hline O que são parasitoses? & $\mathbf{\%}$ & $\mathbf{n}$ \\
\hline Doenças transmitidas por vírus & 4,19 & 6 \\
\hline Doenças transmitidas por bactérias & 0,00 & 0 \\
\hline Doenças transmitidas por parasitos & 77,62 & 111 \\
\hline Doenças transmitidas por fungos & 1,40 & 2 \\
\hline Não desejo responder & 16,79 & 24 \\
\hline Você acha que a administração pública pode conter adisseminação dos mosquitos? & $\mathbf{\%}$ & $\mathbf{n}$ \\
\hline Sim & 50,35 & 72 \\
\hline Não & 16,78 & 24 \\
\hline Não desejo responder & 32,87 & 47 \\
\hline O país pode contar com você para combater a proliferação dos mosquitos? & $\mathbf{\%}$ & $\mathbf{n}$ \\
\hline Sim & 88,81 & 127 \\
\hline Não & 6,29 & 9 \\
\hline Não desejo responder & 4,90 & 7 \\
\hline Se sua casa estiver limpa, pode evitar focos dos mosquitos? & $\mathbf{\%}$ & $\mathbf{n}$ \\
\hline Sim & 39,86 & 57 \\
\hline Não & 55,24 & 79 \\
\hline Não desejo responder & 4,90 & 7 \\
\hline
\end{tabular}

Fonte: Elaboração própria (2017).

Na Tabela 3, foi apresentado um percentual de visitas de agentes de endemias, em casa e na escola. Embora, 67,83\% dos participantes tenham relatado a visita de agentes de endemias em sua residência, 53,14\% responderam que não houveram tais visitas na escola, o que representa um descaso, visto que o trabalho de prevenção e combate aos focos do mosquito Aedes aegypti depende do envolvimento de todos, isto inclui os agentes de combate às endemias. E sobre a presença de recipientes para a criação de mosquitos em casa, a resposta de 53,85\% foi não. Na escola, entretanto, o percentual dos que responderam $\operatorname{sim}$ foi de $60,83 \%$.

Sabe-se que, de modo geral, existem recipientes, descartados incorretamente, que acabam sendo fontes de proliferação da dengue, tanto em ambientes domésticos, quanto escolar. Isso se dá pelo aumento da produção e do consumo de produtos e embalagens não recicláveis, incrementando a produção de lixo e a proliferação de criadouros potenciais (Claro, Tomassini, \& Rosa, 2004).

Com relação as palestras realizadas sobre o tema na escola, conforme a Tabela 3, 90,90\% afirmaram que já assitiram palestras, desse total, 54,54\% relataram que ocorreram apresetações por profissionais de saúde e 48,25\% assistriam palestras ministradas por estudantes de saúde. Tabém foi revelado que apenas 4,19\% receberam informações por parte dos professores do que se evidencia a importância da intervenção através de educação em saúde e ambiental. A escola, como órgão educador, precisa compartilhar as práticas de prevenção contra focos dos mosquitos vetores.

E isso deve ser considerado, já que as informações são geralmente adquiridas pelos estudantes na própria escola, quando há estudos sobre o assunto nas disciplinas de Ciências e Biologia. Esses momentos, porém, não são suficientes para um 
aprofundamento adequado e pode ocorrer também de, às vezes, algumas informações estarem equivocadas ou incompletas, podendo confundir os estudantes (Assis, Pimenta, \& Schall, 2013). Para se completar tal dinâmica - de exposição superficial desse assunto em sala de aula -, palestras, atividades lúdicas e outras estratégias são recomendadas para fortalecer o combate aos mosquitos vetores (Roriz, Peres, \& Ramos, 2016).

Estudos de conhecimento, atitude e prática evidenciam a importância que o acesso às concepções populares tem sobre as estratégias de controle das arboviroses transmitidas por mosquitos vetores (Claro, Tomassini, \& Rosa, 2004).

Embora tenha havido campanhas informativas, que utilizam redes de televisão, rádios, jornais, folhetos, cartazes e palestras comunitárias buscando a colaboração da população para a eliminação dos focos dos mosquitos, já se percebeu que sua eficiência foi limitada (Claro, Tomassini, \& Rosa, 2004).

A vigilância epidemiológica é considerada muitas vezes uma atividade apenas burocrática e não desperta o interesse. Isso só reforça a consciência de que a informação é o ponto de partida para desencadear ações de controle e erradicação (Castro \& Queiroz, 2013).

Tabela 3. Possíveis fatores preventivos contra os mosquitos vetores adotados por alunos de uma escola estadual de Maceió,

Alagoas.

\begin{tabular}{l|c|c}
\hline Já recebeu a visita de agente de endemias em sua residência? & $\mathbf{\%}$ & $\mathbf{n}$ \\
\hline Sim & 67,83 & 97 \\
\hline Não & 27,97 & 40 \\
\hline Não desejo responder & 4,20 & 6 \\
\hline Já recebeu a visita de agente de endemias em sua escola? & $\%$ & $\mathbf{n}$ \\
\hline Sim & 37,76 & 54 \\
\hline Não & 53,14 & 76 \\
\hline Não desejo responder & 9,10 & 13 \\
\hline Em sua residência existem recipientes potenciais para a criação de mosquitos? & $\mathbf{\%}$ & $\mathbf{n}$ \\
\hline Sim & 42,65 & 61 \\
\hline Não & 53,85 & 77 \\
\hline Não desejo responder & 3,50 & 5 \\
\hline Em sua escola existem recipientes potenciais para a criação de mosquitos? & $\mathbf{\%}$ & $\mathbf{n}$ \\
\hline Sim & 60,83 & 87 \\
\hline Não & 32,88 & 47 \\
\hline Não desejo responder & 6,29 & 9 \\
\hline Em sua escola já foi(ram) ralizada(s) palestra(s) sobre estas doenças? & $\%$ & $\mathbf{n}$ \\
\hline Sim & 90,90 & 130 \\
\hline Não & 7,70 & 11 \\
\hline Não desejo responder & 1,40 & 2 \\
\hline
\end{tabular}

Fonte: Elaboração própria (2017).

$\mathrm{Na}$ Tabela 4, quando se analisa a incidência de doenças transmitida por mosquitos, evidencia-se que 59,44\% responderam que não houve ocorrências. Por outro lado, 18,18\% disseram que já tiveram alguma das doenças. Estes, em sua maioria, agora, adotam medidas preventivas para eliminar focos dos mosquitos - uma delas é não deixando água armazenada em depósitos $(25,87 \%)$.

As evidências de casos de doenças causadas por vírus ou parasitos, mesmo sendo em minoria, demonstram a distância entre conhecimento e mudança de comportamento. As situações de risco real de transmissão, indicam, segundo os autores Claro, Tomassini e Rosa (2004), a necessidade de refomulação das ações educativas, enfatizando a participação ativa da 
comunidade.

Existem formas clássicas de se eliminarem criadouros: substituir a água dos vasos de plantas por terra; desobstruir as calhas dos telhados para não acumularem água da chuva; manter sempre tampadas as caixas d'água, cisternas, filtros e barris; não deixar pneus ou quaisquer recipientes que possam acumular água da chuva expostos (Castro \& Queiroz, 2013).

Apenas o conhecimento não é suficiente para eliminar ou conter a disseminação dessas doenças. É fundamental que os indivíduos tenham atitude para praticar tais medidas preventivas e transformar o meio social (Claro, Tomassini, \& Rosa, 2004).

O controle vetorial das doenças transmitidas pelos mosquitos deve partir de uma abordagem eco-bio-social, que necessita da participação dos interessados, inclusive da comunidade local. As atividades devem ser centradas na eliminação de reservatório de água, colocação de tampas em recipientes de proliferação e instalação de telas em janelas e portas (Zara, Santos, Fernandes-Oliveira, Carvalho, \& Coelho, 2016).

Tabela 4. DIPs e medidas de prevenção entre alunos de uma escola estadual de Maceió, Alagoas.

\begin{tabular}{l|c|c}
\hline Você já teve alguma dessas doenças transmitidas por mosquitos? Se sim, qual? & \% & $\mathbf{n}$ \\
\hline Sim & 18,18 & 26 \\
\hline Não & 59,44 & 85 \\
\hline Dengue & 7,69 & 11 \\
\hline Zika & 19,58 & 28 \\
\hline Chikungunya & 5,50 & 8 \\
\hline Febre amarela & 0,00 & 0 \\
\hline Leishmaniose & 0,00 & 0 \\
\hline Malária & 0,00 & 0 \\
\hline Filariose linfática & 0,00 & 0 \\
\hline Não desejo responder & 6,99 & 10 \\
\hline Se sim, quais medidas você passou a adotar após a doença? & $\%$ & $\mathbf{n}$ \\
\hline Não armazenar água em depósitos & 25,87 & 37 \\
\hline Usar mosquiteiro & 5,59 & 8 \\
\hline Fechar as janelas no final da tarde & 12,58 & 18 \\
\hline Usar repelente & 6,29 & 9 \\
\hline Outras & 65,72 & 3 \\
\hline Não desejo responder & 67,81 & 94 \\
\hline
\end{tabular}

Fonte: Elaboração própria (2017).

\subsection{Retorno aos voluntários}

O público deste trabalho compreendeu adolescentes e jovens, uma geração moderna e conectada sedenta por informações; alunos inteligentes, hiperativos e bastante interativos. Nesse sentido, foram realizadas ações como forma de intervenção na comunidade escolar (Quadro 1), no final de cada atividade foi possível notar que os alunos fixaram as informações, o que foi bastante gratificante. 
Quadro 1. Ações de educação em saúde e ambiental realizadas para alunos de uma escola estadual de Maceió, Alagoas.

\begin{tabular}{|c|}
\hline Estratégias de intervenções \\
\hline Confecção de materiais didáticos \\
\hline Palestra sobre dengue \\
\hline Palestra sobre zika \\
\hline Palestra sobre chikungunya \\
\hline Palestra sobre febre amarela \\
\hline Palestra sobre LTA e LVA \\
\hline Palestra sobre malária \\
\hline Palestra sobre filariose linfática \\
\hline Construção da mosquitérica \\
\hline \\
\hline Cinepipoca - Documentário Fiocruz \\
\hline
\end{tabular}

Fonte: Elaboração própria (2017).

Durante as palestras, o conhecimento adquirido pelos alunos ainda em suas residências foi indagado, com o intuito de promover mudanças imediatas no tocante a disseminação das DIPs. Os alunos receberam a intervenção de forma positiva, solicitando para aumentar o tempo de duração das palestras. Estes, estavam extremamente interessados acerca dos mosquitos vetores das DIPs.

Em uma intervenção semelhante, Cordeiro, Silva e Oliveira (2015) apresentaram palestras e distribuíram folders sobre as doenças transmitidas por artrópodes e suas principais formas de prevenção. Do mesmo modo como ocorreu nesta prática, eles notaram interação entre os participantes.

A oficina de construção da mosquitérica ${ }^{\circledR}$ foi realizada pelos próprios alunos utilizando materiais de fácil obtenção (Quadro 2), montando os materiais junto a uma garrafa de Politereftalado de Etileno (PET), seguindo um fluxograma com o passo-a-passo.

Vieira, França, Lima, Souza e Cardim (2017) proporcionaram oportunidade de compreensão e conscientização sobre dengue, realizando um projeto extensionista para estudantes do ensino fundamental, envolvendo apresentações orais, peça teatral, atividades práticas e distribuição de material educativo.

De acordo com Santos, Balbé, Corrêa, Matter e Pillatt (2016), ações multidisciplinares e transdisciplinares devem envolver metodologias lúdicas, revelando e solucionando problemas discutíveis em equipes multiprofissionais.

Quadro 2. Materiais utilizados para confecção da mosquitérica ${ }^{\circledR}$ por alunos de uma escola estadual de Maceió, Alagoas.

\begin{tabular}{|c|}
\hline Descrição dos materiais \\
\hline Garrafa PET \\
\hline Lixa de madeira \\
\hline Tule \\
\hline Tesoura \\
\hline Arroz \\
\hline Água \\
\hline Fita adesiva \\
\hline
\end{tabular}

Fonte: Faperj (2013).

Durante o momento de exibição do documentário "Aedes aegypti e Aedes albopictus - Uma ameaça nos Trópicos”, as salas de aula se transformaram em cinema: as imagens foram projetadas no fundo da sala, com direito à pipoca de milho. Os 
estudantes puderam acompanhar como ocorre a dispersão das espécies pelos continentes de clima tropical e subtropical, bem como, as características morfológicas e os hábitos alimentares dos mosquitos.

Corroborando com o disposto, Souza Neto (2016), reconhece que as formas didáticas audiovisuais de educação em saúde se demonstram como uma possibilidade instigante de expansão de uma lógica preventiva. A ação intervencionista também diminui a distância entre a academia e a comunidade.

\section{Conclusão}

Verifica-se que a maiorias dos alunos reconhecem a necessidade de se ter atitudes preventivas contra os mosquitos vetores, adotando em suas práticas diárias medidas como não acumular água e destinar o lixo para o local apropriado. Grande parte dos estudantes possui algum conhecimento quanto as doenças provocadas pelos mosquitos vetores que são os transmissores de vírus e parasitos.

Não obstante desse contexto, a escola possui uma função essencial quanto a transmissão do conhecimento a respeito das medidas preventivas, pois apesar do empenho para eliminação dos criadouros de mosquitos verificado no trabalho, uma pequena parcela não demonstra o interesse necessário, enfatizando a importância de incluir esta temática no ambiente escolar para que possa ser aplicada no cotidiano dos alunos e assim promover a melhoria da saúde pública.

Os resultados apontam para a necessidade de se desenvolver atividades educativas com aspectos relativos à concepção de promoção da saúde, com públicos de diferentes locais e idades, dentro e fora do ambiente escolar, que possam contribuir com a problematização das doenças infecciosas transmitidas por mosquitos vetores bem como o estímulo à ação popular no planejamento e nas tomadas de decisões com o intuito de desencadear mudanças de hábitos individuais relacionadas as formas de prevenção.

\section{Referências}

Aguiar-Santos, A. M, Medeiros, Z., Bonfim, C., Rocha, A. C., Brandão, E., Miranda, T., Oliveira, P., \& Sarinho, E. S. C. (2013). Epidemiological assessment of neglected diseases in children: lymphatic filariasis and soil-transmitted helminthiasis. Jornal de Pediatria, 89(3), 250-255. http://doi.org/10.1016/j.jped.2012.11.003

Alvarenga, D. G., Escalda, P. M. F., Costa, A. S. V., \& Monreal, M. T. F. D. (2010). Leishmaniose visceral: estudo retrospectivo de fatores associados à letalidade. Revista da Sociedade Brasileira de Medicina Tropical, 43(2), 194-197. http://doi.org/10.1590/S0037-86822010000200017

Anuário Estatístico de Alagoas. (2017). Alagoas em dados e informações. http://dados.al.gov.br/dataset/anuario-estatisticodo-estado-de-alagoas

Assis, S. S., Pimenta, D. N., \& Schall, V. T. (2013). A dengue nos livros didáticos de ciência e biologia indicados pelo programa nacional do didático. Ciência \& Educação, 19(3), 633-656. http://doi.org/10.1590/S1516-73132013000300009

Assunção, M. L., \& Aguiar, A. M. M. (2014). Perfil clínico-epidemiológico da dengue no município de Juscimeira - MT. Revista de Epidemiologia e Controle de Infecção, 4(4), 249-253. https://www.redalyc.org/articulo.oa?id=570463901006

Beinner, M. A., Norton, R. C., \& Lamounier, J. A. (2006). Prevalência da infecção por parasitas intestinais em crianças pré-escolares de distritos rurais do município de Diamantina, Minas Gerais. Revista Médica de Minas Gerais, 16(2), 84-87. http://www.rmmg.org/artigo/detalhes/259

Borges, B. K. A., Silva, J. A., Haddad, J. P. A., Moreira, E. C., Magalhães, D. F., Ribeiro, L. M. L., \& Fiúza, V. O. P. (2009). Presença de animais associada ao risco de transmissão da leishmaniose visceral em humanos em Belo Horizonte, Minas Gerais. Arquivo Brasileiro de Medicina Veterinária e Zootecnia, 61(5), 1035-1043. http://doi.org/10.1590/S0102-09352009000500004

Borges, E. A., \& Oliveira, M. A. (2011, Maio). Educação ambiental com ênfase no consumo consciente e o descarte de resíduos - uma experiência da educação formal. Anais do Simpósio de Educação Ambiental e Transdisciplinaridade, Goiânia, GO, Brasil, II. https://files.cercomp.ufg.br/weby/up/52/o/31_Consumo_consciente.pdf

Braga, C., Ximenes, R. A. A., Albuquerque, M. F. P. M., Souza, W. V., Miranda, J., Brayner, F., Alves, L., Silva, L., \& Dourado, I. (2001). Avaliação de indicador sócio-ambiental utilizado no rastreamento de áreas de transmissão de filariose linfática em espaços urbanos. Cadernos de Saúde Pública, 17(5), 1211-1218. http://doi.org/10.1590/S0102-311X2001000500021

Braga, E. M., \& Fontes, C. J. F. (2010). Plasmodium: malária. In: Neves, D. P., Melo, A. L., Linardi, P. M., \& Vitor, R. W. A. (Orgs.). Parasitologia humana (11a ed., chap. 17, pp. 143-161). São Paulo: Editora Atheneu.

Castro, E. A., \& Queiroz, P. R. (2013, Novembro). O vírus da dengue no brasil e as medidas de prevenção, controle e erradicação. Anais da Mostra de Produção Científica PósGraduação Lato Sensu. Goiânia, GO, Brasil, 8. 
Claro, L. B. L., Tomassini, H. C. B., \& Rosa, M. L. G. (2004). Prevenção e controle do dengue: uma revisão de estudos sobre conhecimentos, crenças e práticas da população. Cadernos de Saúde Pública, 20(6), 1447-1457. http://doi.org/10.1590/S0102-311X2004000600002

Confalonieri, U. E. C. (2003). Variabilidade climática, vulnerabilidade social e saúde no Brasil. Terra Livre, I(20), $193-204$.

Cordeiro, L. L. L. R., Silva, A. C., \& Oliveira, E. L. R. (2015). Programa de educação para prevenção e controle de artrópodes transmissores de doenças no município de Viçosa, Alagoas. Em Extensão, 14(2), 186-200. http://doi.org/10.14393/REE-v14n22015_rel06

Fundação de Amparo à Pesquisa do Estado do Rio de Janeiro - Faperj. (2013). Armadilha letal para mosquitos, temperada com atitude de civilidade. http://www.faperj.br/downloads/mosquiterica.pdf

Gil, A. C. (2008). Métodos e técnicas de pesquisa social (6a ed.). São Paulo: Atlas. https://ayanrafael.files.wordpress.com/2011/08/gil-a-c-mc3a9todos-etc3a9cnicas-de-pesquisa-social.pdf

Laboissière, P. (2016). Aedes aegypti já se tornou mosquito dosméstico, alerta epidemiologista. https://agenciabrasil.ebc.com.br/geral/noticia/2016-02/aedesja-se-tornou-mosquito-domestico-alerta-epidemiologista

Lesser, J., \& Kitron, U. (2016). A geografia social do zika no Brasil. Estudos Avançados, 30(88), 167-175. http://doi.org/10.1590/s0103-40142016.30880012

Lobo, K. S., Bezerra, J. M. T., Brito, L. M. O., Silva, J. S., \& Pinheiro, V. C. S. (2013). Conhecimentos de estudantes sobre Leishmaniose Visceral em escolas públicas de Caxias, Maranhão, Brasil. Ciência \& Saúde Coletiva, 18(8), 2295-2300. http://doi.org/10.1590/S1413-81232013000800013

Ludke, M., \& Andre, M. E. D. A. (2013). Pesquisas em educação: uma abordagem qualitativa. São Paulo: E.P.U.

Mascheretti, M., Tengan, C. H., Sato, H. K., Suzuki, A., Souza, R. P., Maeda, M., Brasil, R., Pereira, M., Tubaki, R. M., Wanderley, D. M. V., Fortaleza, C. M. C. B., \& Ribeiro, A. F. (2013). Febre amarela silvestre: reemergência de transmissão no estado de São Paulo, Brazil, 2009. Revista de Saúde Pública, 47(5), 881-889. https://doi.org/10.1590/S0034-8910.2013047004341

Ministério da Saúde. (2010). Doenças infecciosas e parasitárias: guia de bolso (8a ed.). Secretaria de Vigilância da Saúde. Brasília, DF: Ministério da Saúde. https://bvsms.saude.gov.br/bvs/publicacoes/doencas_infecciosas_parasitaria_guia_bolso.pdf

Oliveira, K. K. D., Fernandes, A. P. N. L., \& Moura, S. G. F. (2012). Perfil de morbidade por patologias infecto-contagiosas entre crianças de 0 a 12 anos. Fiep Bulletin On-line, 82. http://www.fiepbulletin.net/index.php/fiepbulletin/article/view/2303

Pedrosa, H. F. S. (2008). Avaliação do impacto da aplicação das diretrizes do planto diretor de Maceió sobre o escoamento superficial. Dissertação de mestrado, Universidade Federal de Alagoas, Maceió, AL, Brasil. http://www.repositorio.ufal.br/jspui/handle/riufal/5252

Pereira, A. S., Shitsuka, D. M., Parreira, F. J., \& Shitsuka, R. (2018). Metodologia da pesquisa científica. Santa Maria: UFSM/ NTE. https://www.ufsm.br/app/uploads/sites/358/2019/02/Metodologia-da-Pesquisa-Cientifica_final.pdf

Queiroz, M. F. M., Varjão, J. R., Moraes, S. C., \& Salcedo, G. E. (2012). Analysis of sandflies (Diptera: Psychodidae) in Barra do Garças, state of Mato Grosso, Brazil, and the influence of environmental variables on the vector density of Lutzomyia longipalpis (Lutz \& Neiva, 1912). Revista da Sociedade Brasileira de Medicina Tropical, 45(3), 313-317. http://doi.org/10.1590/S0037-86822012000300007

Ribeiro, J. W., \& Rooke, J. M. S. (2010). Saneamento básico e sua relação com o meio ambiente e a saúde pública. Trabalho de Conclusão de Curso de especialização, Universidade Federal de Juiz de Fora, Juiz de Fora, MG, Brasil. https://www.ufjf.br/analiseambiental/files/2009/11/TCCSaneamentoeSa\%C3\%BAde.pdf

Roriz, P. A. C., Peres, W. V., \& Ramos, R. R. (2016). Percepção de estudantes de $9^{\circ}$ ano sobre dengue, zika e chikungunya. Nexus Revista de Extensão do IFAM, 2(2), 93-100. http://nexus.ifam.edu.br/nexus/index.php?journal=Nexus\&page=article\&op=view\&path\%5B $\% 5 \mathrm{D}=104 \& p a t h \% 5 \mathrm{~B} \% 5 \mathrm{D}=70$

Santos, F. P., Balbé, A. S., Corrêa, F. G., Matter, P. S., \& Pillatt, A. P. (2016). Convergência de saberes multidisciplinares: teorias e práticas na práxis em saúde na escola. Anais do Salão do Conhecimento Unijuí, Ijuí, https://www.publicacoeseventos.unijui.edu.br/index.php/salaoconhecimento/article/view/6552

Secretaria de Estado da Saúde de Alagoas - Sesau. (2017). http://saude.al.gov.br/

Silva, S. A., Gama, J. A. S., Callado, N. H., \& Souza, V. C. B. (2017). Saneamento básico e saúde pública na Bacia Hidrográfica do Riacho Reginaldo em Maceió, Alagoas. Engenharia Sanitária e Ambiental, 22(4), 699-709. http://doi.org/10.1590/S1413-41522017146971

Souza Neto, V. L. (2016). Elaboração e implementação de ferramenta educativa com ênfase na dengue, zika e chikungunya: relato de experiência. Revista Extendere, 4(1), 9-18. http://periodicos.uern.br/index.php/extendere/article/view/2033

Valverde, R. (2016). Doenças negligenciadas. http://agencia.fiocruz.br/doen\%C3\%A7asnegligenciadas

Vieira, S. N. S., França, L. S., Lima, J. J. P., Souza, F. S., \& Cardim, S. Q. (2017). Educação em saúde e o combate à dengue: um relato de experiência. Revista de Enfermagem UFPE on line, 11(5), 2227-2230. https://doi.org/10.5205/1981-8963-v11i5a23379p2227-2230-2017

Zara, A. L. S. A., Santos, S. M., Fernandes-Oliveira, E. S., Carvalho, R. G., \& Coelho, G. E. (2016). Estratégias de controle do Aedes aegypti: uma revisão. Epidemiologia e Serviços Saúde, 25(2), 391-404. http://doi.org/10.5123/S1679-49742016000200017 